\title{
Direct Numerical Simulation of Shock/Boundary Layer Interaction over Surface Roughness Using the High-Oder FR/CPR-LLAV Method
}

\author{
Meilin $\mathrm{Yu}^{1}(\bowtie)$ \\ Department of Mechanical Engineering \\ University of Maryland, Baltimore County (UMBC), Baltimore, MD 21250
}

\begin{abstract}
A recently developed high-order accurate flux reconstruction/correction procedure via reconstruction (FR/CPR) method equipped with localized Laplacian artificial viscosity (LLAV) shock-capturing capability is used to simulate both two dimensional (2D) and three dimensional (3D) shock-boundary layer interaction (SBLI) at different Reynolds numbers with and without wall roughness. It is found that the method can effectively and sharply resolve both shock and boundary-layer separation features. From the SBLI simulation results, it is observed that surface roughness can change the interaction between the shock waves and the boundary layers. As a result, the surface heat transfer process can be substantially modified.
\end{abstract}

\section{Introduction}

During the past half-century, remarkable progress has been achieved in both experimental and numerical research on hypersonic flow, as seen in the recent comprehensive reviews on shock-boundary layer interaction (SBLI) and shock-turbulence interaction [1, 2, 3, 4]. However, many physical problems have yet to be explored more fully due to the multi-disciplinary nature of hypersonic flow. One example is the shock-induced separation in boundary layers. Shock-induced separation can dramatically affect the aerodynamic performance of flying vehicles, causing increased drag and loss of lift. It can also generate low-frequency separation bubbles, which may induce fatigue of structures. The intermittent feature of the separation bubbles can substantially change the surface heat transfer process, e.g., facilitating extremely high temperature spots on the structure surface, thus posing great challenge on thermal protection. Therefore, it is of great importance to have clear understanding on the underlying physics behind the shock-induced boundary layer separation.

Numerical simulation provides a cost-effective way to study these hypersonic flow phenomena. But as has been realized by many researchers $[5,6]$, the surface heat flux prediction can be completely wrong due to the insufficient resolution of flow near strong shock waves. As a result, there exists practical need in high-fidelity numerical tools for accurately predicting the physical processes involved. Since the key flow features in shock-induced separation include both flow discontinuity and vortices, the numerical methods should have the following capabilities simultaneously. First, the method needs to be capable of robustly and efficiently stabilize flow discontinuity without contaminating smooth flow structures elsewhere. Secondly, the method needs to be capable of accurately predicting the boundary layer separation at the existence of shock waves, including separation point, fluctuation frequency, and impinging shock position and deflective angle.

\footnotetext{
${ }^{1}$ Assistant Professor, Department of Mechanical Engineering, AIAA Member, email: mlyu@umbc.edu
} 
The flux reconstruction/correction procedure via reconstruction (FR/CPR) method [7] equipped with shock-capturing capability $[8,9,10]$, is used in current study to numerically investigate shock-induced separation. It is noted that the FR/CPR method unifies many existing high-order CFD methods such as discontinuous Galerkin (DG) [11, 12], spectral volume (SV) [13] and spectral difference (SD) [14]. From the work in [15], it is also demonstrated that comparing with quadrature-based DG (QDG), nodal DG (NDG), and SD, the FR/CPR formulation is the most cost-effective scheme on quadrilateral mesh due to its 1D features. As reported by Candler et al. [4], the DG type methods have the potential to conquer the challenges posed by hypersonic simulation, and can take full advantage of the rocketing computation powers nowadays. Therefore, a recently developed high-order FR/CPR-DG method for hypersonic flow simulation $[16,10,17]$ will be further improved for the current study.

The reminder of the paper is organized as follows. In Section II, the numerical methods, including the FR/CPR algorithm and the localized Laplacian artificial viscosity methods, are introduced. The numerical results and discussion are then presented in Section III. Specifically, the numerical results of shockboundary layer interaction at different Reynolds numbers with and without wall roughness will be discussed there. Section IV concludes the paper.

\section{Numerical Methods}

Consider the unsteady compressible Navier-Stokes (N-S) equations in conservation form,

$$
\frac{\partial Q}{\partial t}+\nabla \cdot \boldsymbol{F}(Q)=0
$$

defined on $\Omega \times[0, T]$ with spatial domain $\Omega$ bounded by $\partial \Omega$, where $Q$ is the vector of conserved variables, and $\boldsymbol{F}=(f, g, h)$ is the flux vector including both the inviscid and viscous components.

To achieve an efficient implementation, the conservation law is usually transformed from the physical domain $(x, y, z)$ into the computational domain $(\xi, \eta, \zeta)=[-1,1]^{3}$. Define the area vectors

$$
\begin{aligned}
\boldsymbol{a}^{1} & =\left(\left|\begin{array}{ll}
y_{\eta} & y_{\zeta} \\
z_{\eta} & z_{\zeta}
\end{array}\right|,-\left|\begin{array}{ll}
x_{\eta} & x_{\zeta} \\
z_{\eta} & z_{\zeta}
\end{array}\right|,\left|\begin{array}{ll}
x_{\eta} & x_{\zeta} \\
y_{\eta} & y_{\zeta}
\end{array}\right|\right), \\
\boldsymbol{a}^{2} & =\left(-\left|\begin{array}{ll}
y_{\xi} & y_{\zeta} \\
z_{\xi} & z_{\zeta}
\end{array}\right|,\left|\begin{array}{cc}
x_{\xi} & x_{\zeta} \\
z_{\xi} & z_{\zeta}
\end{array}\right|,-\left|\begin{array}{ll}
x_{\xi} & x_{\zeta} \\
y_{\xi} & y_{\zeta}
\end{array}\right|\right), \\
\boldsymbol{a}^{3} & =\left(\left|\begin{array}{ll}
y_{\xi} & y_{\eta} \\
z_{\xi} & z_{\eta}
\end{array}\right|,-\left|\begin{array}{ll}
x_{\xi} & x_{\eta} \\
z_{\xi} & z_{\eta}
\end{array}\right|,\left|\begin{array}{ll}
x_{\xi} & x_{\eta} \\
y_{\xi} & y_{\eta}
\end{array}\right|\right),
\end{aligned}
$$

and the volume

$$
\tau=\left|\begin{array}{lll}
x_{\xi} & x_{\eta} & x_{\zeta} \\
y_{\xi} & y_{\eta} & y_{\zeta} \\
z_{\xi} & z_{\eta} & z_{\zeta}
\end{array}\right|
$$

where $x_{\xi}, x_{\eta}, x_{\zeta}, y_{\xi}, y_{\eta}, y_{\zeta}, z_{\xi}, z_{\eta}$ and $z_{\zeta}$ are metrics of the coordinate transformation. Then the transformed equation of Eq. (1) takes the following form

where

$$
\frac{\partial \tilde{Q}}{\partial t}+\frac{\partial \tilde{f}}{\partial \xi}+\frac{\partial \tilde{g}}{\partial \eta}+\frac{\partial \tilde{h}}{\partial \zeta}=0
$$

$$
\tilde{Q}=\tau Q,(\tilde{f}, \tilde{g}, \tilde{h})=\left(\boldsymbol{a}^{1} \cdot \boldsymbol{F}, \boldsymbol{a}^{2} \cdot \boldsymbol{F}, \boldsymbol{a}^{3} \cdot \boldsymbol{F}\right) .
$$

It is assumed that the physical domain $\Omega$ is partitioned into $\mathrm{N}$ non-overlapping elements $\Omega^{i}$. Note that Eq. (1) holds on each $\Omega^{i}$. When $\Omega^{i}$ is transformed into the corresponding standard element $\Omega_{s}$, Eq. (4) holds on $\Omega_{s}$. 


\section{II.1. Flux Reconstruction/Correction Procedure via Reconstruction (FR/CPR) Method}

In FR/CPR, the flux terms in Eq. (4), i.e., $\tilde{f}, \tilde{g}$ and $\tilde{h}$, are treated as a combination of local fluxes, namely, $\tilde{f}^{L o c}, \tilde{g}^{L O C}$ and $\tilde{h}^{L o c}$, and correction fluxes, namely, $\tilde{f}^{C o r}, \tilde{g}^{\text {Cor }}$ and $\tilde{h}^{C o r}$. Mathematically, this can be written as

$$
\left\{\begin{array}{l}
\tilde{f}(\xi, \eta, \zeta)=\tilde{f}^{L o c}(\xi, \eta, \zeta)+\tilde{f}^{\operatorname{Cor}}(\xi, \eta, \zeta) \\
\tilde{g}(\xi, \eta, \zeta)=\tilde{g}^{\operatorname{Loc}}(\xi, \eta, \zeta)+\tilde{g}^{\operatorname{Cor}}(\xi, \eta, \zeta) . \\
\tilde{h}(\xi, \eta, \zeta)=\tilde{h}^{L o c}(\xi, \eta, \zeta)+\tilde{h}^{\operatorname{Cor}}(\xi, \eta, \zeta)
\end{array}\right.
$$

Local fluxes are constructed using only flow information within a specific element. Therefore, local fluxes are element-wise continuous, and have jumps on element boundaries. To ensure conservation and numerical stability, common or numerical fluxes, i.e., $\tilde{f}^{N u m}, \tilde{g}^{\text {Num }}$ and $\tilde{h}^{\text {Num }}$ in current context, are reconstructed on element boundaries using local flow information via Riemann solvers [18] for the inviscid fluxes or via the second Bassi-Rebay (BR2) approach [19] for the viscous fluxes. The numerical fluxes are then used to correct the local fluxes, and forms the correction fluxes $\tilde{f}^{\text {Cor }}, \tilde{g}^{\text {Cor }}$ and $\tilde{h}^{\text {Cor }}$. This procedure for a quadrilateral element can be expressed as

$$
\begin{aligned}
& \tilde{f}^{\operatorname{Cor}}(\xi, \eta, \zeta)=\left(\tilde{f}_{L}^{N u m}(\eta, \zeta)-\tilde{f}^{L o c}(-1, \eta, \zeta)\right) G_{L}(\xi)+\left(\tilde{f}_{R}^{N u m}(\eta, \zeta)-\tilde{f}^{L o c}(1, \eta, \zeta)\right) G_{R}(\xi), \\
& \tilde{g}^{\operatorname{Cor}}(\xi, \eta, \zeta)=\left(\tilde{g}_{L}^{N u m}(\xi, \zeta)-\tilde{g}^{L o c}(\xi,-1, \zeta)\right) G_{L}(\eta)+\left(\tilde{g}_{R}^{N u m}(\xi, \zeta)-\tilde{g}^{L o c}(\xi, 1, \zeta)\right) G_{R}(\eta), \\
& \tilde{h}^{\operatorname{Cor}}(\xi, \eta, \zeta)=\left(\tilde{h}_{L}^{\text {Num }}(\xi, \eta)-\tilde{h}^{L o c}(\xi, \eta,-1)\right) G_{L}(\zeta)+\left(\tilde{h}_{R}^{N u m}(\xi, \eta)-\tilde{h}^{L o c}(\xi, \eta, 1)\right) G_{R}(\zeta) .
\end{aligned}
$$

Herein, the subscripts ' $L$ ' and ' $R$ ' stand for the left and right boundaries of an element in a dimension-bydimension sense. $G_{L}$ and $G_{R}$ are correction functions, which map the differences between numerical fluxes and local fluxes on the boundaries into the entire element. Different choices of the correction functions $G_{L}$ and $G_{R}$ result in different numerical schemes, see Refs. [20, 21]. In the present study, $G_{L}$ and $G_{R}$ are chosen as right and left Radau polynomials, which recovers the standard DG scheme.

On substituting Eq. (6) into Eq. (4), the governing equations on each element $\Omega^{s}$ then read

$$
\begin{gathered}
\frac{\partial \tilde{Q}}{\partial t}+\left(\frac{\partial \tilde{f}^{L o c}}{\partial \xi}+\frac{\partial \tilde{g}^{L o c}}{\partial \eta}+\frac{\partial \tilde{h}^{L o c}}{\partial \zeta}\right)+\left(\frac{\partial \tilde{f}^{C o r}}{\partial \xi}+\frac{\partial \tilde{g}^{C o r}}{\partial \eta}+\frac{\partial \tilde{h}^{C o r}}{\partial \zeta}\right) \\
=\frac{\partial \tilde{Q}}{\partial t}+\frac{\partial \tilde{f}^{L o c}}{\partial \xi}+\frac{\partial \tilde{g}^{L o c}}{\partial \eta}+\frac{\partial \tilde{h}^{L o c}}{\partial \zeta}+\tilde{\delta}^{C o r}=0 .
\end{gathered}
$$

Herein, $\tilde{\delta}^{\text {Cor }}=\tau \delta^{\text {Cor }}=\partial \tilde{f}^{\text {Cor }} / \partial \xi+\partial \tilde{g}^{\text {Cor }} / \partial \eta+\partial \tilde{h}^{\text {Cor }} / \partial \zeta$ is named as the correction field.

In FR/CPR, the differential form of the conservation law, i.e., Eq. (8), is solved numerically on the standard element with interior solution points and interface (element boundary) flux points. Two sets of allocation of solution points and interface flux points for quadrilateral elements are displayed in Fig. 1. In Figure 1(a), Lobatto points are selected as solution points, and the flux points coincide with the solution points on element boundaries. In Figure 1(b), Gauss points are selected as solution points, and the corresponding flux points are marked on the element boundaries. 


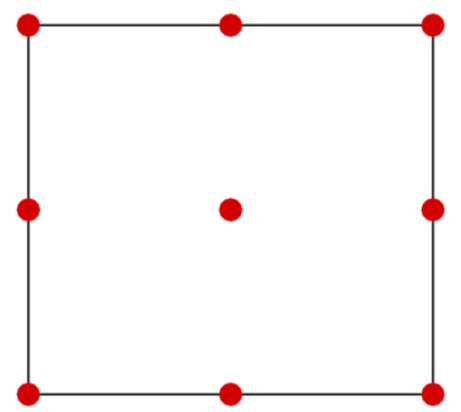

(a)

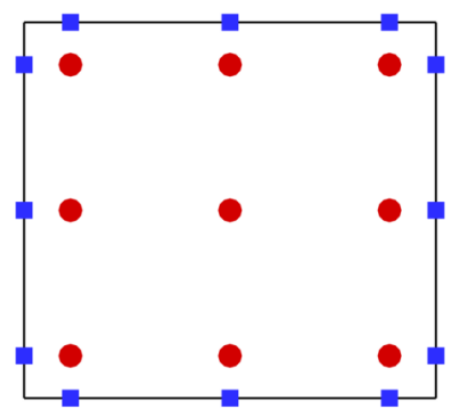

(b)

Figure 1. Two types of collocation of the solution points (red dots) and interface flux points (blue squares) on quadrilateral elements. (a) Solution points: Legendre-Gauss-Lobatto points; Interface flux points (coincided with solution points): Legendre-Gauss-Lobatto points; (b) Solution points: Gauss-Legendre points; Interface flux points: Gauss -Legendre points.

First of all, we approximate the exact solution of the conservation law using an element-wise continuous polynomial $\tilde{Q}_{h}=\tau Q_{h} \in \mathbb{Q}^{k}\left(\Omega_{s}\right)$, where $\mathbb{Q}^{k}\left(\Omega_{s}\right)$ is the space of tensor product of polynomials of degree at most $\mathrm{k}$ in each variable defined on $\Omega_{\mathrm{s}}$. The local fluxes $\tilde{f}^{L o c}, \tilde{g}^{L o c}$ and $\tilde{h}^{L o c}$ are assumed to sit in the same space as $\tilde{Q}_{h}$, expressing as $\tilde{f}^{L o c} \in \mathbb{Q}^{k}\left(\Omega_{S}\right), \tilde{g}^{L o c} \in \mathbb{Q}^{k}\left(\Omega_{S}\right)$ and $\tilde{h}^{L o c} \in \mathbb{Q}^{k}\left(\Omega_{S}\right)$. The derivative of the local fluxes can be calculated using the Lagrange polynomial (LP) approach. Specifically, $\tilde{f}^{L o c}, \tilde{g}^{L o c}$ and $\tilde{h}^{L o c}$ can be expanded with the linearly independent basis consisting of Lagrange polynomials. The derivatives of the local fluxes are then expressed as

$$
\begin{aligned}
& \frac{\partial \tilde{f}^{L o c}}{\partial \xi}=\sum_{j \in S P} \frac{\partial}{\partial \xi} L_{j}(\xi, \eta, \zeta) \tilde{f}_{j}^{L o c}, \\
& \frac{\partial \tilde{g}^{L o c}}{\partial \eta}=\sum_{j \in S P} \frac{\partial}{\partial \eta} L_{j}(\xi, \eta, \zeta) \tilde{g}_{j}^{L o c}, \\
& \frac{\partial \tilde{h}^{L o c}}{\partial \zeta}=\sum_{j \in S P} \frac{\partial}{\partial \zeta} L_{j}(\xi, \eta, \zeta) \tilde{h}_{j}^{L o c} .
\end{aligned}
$$

where ' $S P$ ' stands for the set of solution points. Note that for a quadrilateral element, $L_{j}$ is a tensor product of two 1D Lagrange polynomials, $l_{j}(\xi), l_{j}(\eta)$ and $l_{j}(\zeta)$, associated with the solution point ' $j$ '.

To ensure the order of accuracy, $\tilde{\delta}^{\text {Cor }}$ needs to be approximated by the same space as $\tilde{Q}_{h}$, which states $\tilde{\delta}^{\text {Cor }} \in \mathbb{Q}^{k}\left(\Omega_{S}\right)$. According to Eq. (7), the correction functions $G_{L}$ and $G_{R}$ must belong to the polynomial space $P^{k+1}\left(\partial \Omega_{\mathrm{s}}\right)$. To recover the DG scheme, $G_{L}$ and $G_{R}$ are right and left Radau polynomials written as

$$
G_{L, k+1}=R_{R, k+1}=\frac{(-1)^{k+1}}{2}\left(L_{k+1}-L_{k}\right), G_{R, k+1}=R_{L, k+1}=\frac{1}{2}\left(L_{k+1}+L_{k}\right),
$$

where $R_{R, k+1}$ and $R_{L, k+1}$ indicate the right and left Radau polynomials of degree $k+1$ respectively, and $L_{k+1}$ and $L_{k}$ are Legendre polynomials of degree $k+1$ and $k$ respectively.

On regular linear elements, the metrics $x_{\xi}, x_{\eta}, x_{\zeta}, y_{\xi}, y_{\eta}, y_{\zeta}, z_{\xi}, z_{\eta}$ and $z_{\zeta}$ are constant. As reported in Ref. [15], the approximation $\widetilde{Q}_{h} \in \mathbb{Q}^{k}\left(\Omega_{S}\right)$ is equivalent to $Q_{h} \in \mathbb{Q}^{k}\left(\Omega_{S}\right)$. Similar arguments work for $f^{L O c}, g^{L O C}, h^{L O C}, \delta^{C o r}, \tilde{f}^{L O c}, \tilde{g}^{L O c}, \tilde{h}^{L O c}$ and $\tilde{\delta}^{C o r}$. However, this does not hold on curved elements. In order to enhance the accuracy of the FR/CPR method on curved elements, an effective way, as proposed 
in Ref. [15], is to assume that $Q_{h}, f^{L o c}, g^{L o c}, h^{L o c}$ and $\tilde{\delta}^{L o c} \in \mathbb{Q}^{k}\left(\Omega_{S}\right)$. Under this assumption, Eq. (8) can reformulated as

$$
\frac{\partial Q}{\partial t}+\frac{\partial f^{L o c}}{\partial x}+\frac{\partial g^{L o c}}{\partial y}+\frac{\partial h^{L o c}}{\partial z}+\frac{\tilde{\delta}^{C o r}}{\tau}=\frac{\partial Q}{\partial t}+\frac{\partial f^{L o c}}{\partial x}+\frac{\partial g^{L o c}}{\partial y}+\frac{\partial h^{L o c}}{\partial z}+\delta^{C o r}=0 .
$$

with

$$
\delta^{\operatorname{Cor}}=\frac{1}{\tau}\left(\frac{\partial \tilde{f}^{\text {Cor }}}{\partial \xi}+\frac{\partial \tilde{g}^{\text {Cor }}}{\partial \eta}\right)
$$

Note that the chain rule is used to calculate the derivative of local fluxes in Eq. (11), which is expressed as

$$
\begin{aligned}
& \frac{\partial f^{L o c}}{\partial x}=\frac{\partial f^{L o c}}{\partial \xi} \xi_{x}+\frac{\partial f^{L o c}}{\partial \eta} \eta_{x}+\frac{\partial f^{L o c}}{\partial \zeta} \zeta_{x}, \\
& \frac{\partial g^{L o c}}{\partial y}=\frac{\partial g^{L o c}}{\partial \xi} \xi_{y}+\frac{\partial g^{L o c}}{\partial \eta} \eta_{y}+\frac{\partial g^{L o c}}{\partial \zeta} \zeta_{y}, \\
& \frac{\partial h^{L o c}}{\partial z}=\frac{\partial h^{L o c}}{\partial \xi} \xi_{z}+\frac{\partial h^{L o c}}{\partial \eta} \eta_{z}+\frac{\partial h^{L o c}}{\partial \zeta} \zeta_{z} .
\end{aligned}
$$

This completes the setup of the FR/CPR method on general quadrilateral elements. Readers are referred to Refs. [20, 22, 23, 24, 21] for more information on this method.

\section{II.2. Localized Laplacian Artificial Viscosity (LLAV)}

As concluded from the $1^{\text {st }}$ to $3^{\text {rd }}$ high-order CFD workshop [25], robust and accuracy preserving shock capturing is still one of the challenges in the development of high-order CFD methods. Herein, we introduce a Laplacian localized artificial viscosity method, which can suppress the spurious oscillation in the vicinity of flow discontinuity but not contaminate the smooth flow features elsewhere. In this context, the Navier-Stokes equations are augmented with Laplacian diffusion terms. Generally, for 3D problems, the Laplacian diffusion terms in each element can be written as

$$
\nabla \cdot \boldsymbol{F}^{a v}(\nabla Q)=\frac{\partial}{\partial x}\left(\varepsilon_{e, x} \frac{\partial Q}{\partial x}\right)+\frac{\partial}{\partial y}\left(\varepsilon_{e, y} \frac{\partial Q}{\partial y}\right)+\frac{\partial}{\partial z}\left(\varepsilon_{e, z} \frac{\partial Q}{\partial z}\right),
$$

where $\varepsilon_{e, x}, \varepsilon_{e, y}$ and $\varepsilon_{e, z}$ are artificial viscosity in $x, y$ and $z$ directions. A resolution-based smoothness indicator, which can distinguish flow discontinuity, is used to localize the artificial viscosity near shocks in the flow field.

Generally, a smooth variation of the element-wise artificial viscosity $\varepsilon_{e}$ can reconstructed as follows,

$$
\varepsilon_{e}=\left\{\begin{array}{cl}
\frac{\varepsilon_{0}}{2}\left(1+\sin \frac{\pi\left(S_{e}-S_{0}\right)}{2 \kappa}\right) & \text { if } S_{e}<S_{0}-\kappa \\
\varepsilon_{0} & \text { if } S_{0}-\kappa \leq S_{e} \leq S_{0}+\kappa \\
\text { if } S_{e}>S_{0}+\kappa .
\end{array}\right.
$$

Herein, $S_{0}$ is the estimated value of the smoothness indicator $S_{e}$ of the flow field, $\kappa$ is a dissipation spectra control parameter, and $\varepsilon_{0}$ is the maximum value of artificial viscosity. Based on the Refs. [10, $16], \varepsilon_{0}$ can be modelled as

$$
\varepsilon_{0}=\left(-\frac{\Delta \xi_{\max }}{P e}+\frac{2}{P e}\right) \cdot\left|\Delta \vec{x} \cdot \frac{\nabla \rho}{|\nabla \rho|}\right| \cdot U_{r e f}
$$


where $P e$ is the diffusion Péclet number; $\Delta \xi_{\max }$, scaled in [0,1], is the maximum distance between two adjacent quadrature points in a standard $1 \mathrm{D}$ element; $\Delta \vec{x}$ is the grid size vector; $\nabla \rho$ is the density gradient; and $U_{\text {ref }}$ is the reference velocity.

In order to eliminate the undesirable artificial viscosity on expansion waves, a dilatation condition proposed in Ref. [26], is added to the smoothness criteria, which states "if the velocity divergence $\nabla \cdot \vec{u}$ is larger than zero, then the flow field is treated as smooth." For implementation, the artificial viscosity $\varepsilon_{e}$ is limited as follows:

$$
\varepsilon_{e}^{\lim }=\left\{\begin{array}{ll}
0 & \text { if } \nabla \cdot u>0 \\
\varepsilon_{e} & \text { if } \nabla \cdot u \leq 0
\end{array} .\right.
$$

More modeling details regarding the Laplacian localized artificial viscosity can be found in Refs. $[10,16]$.

\section{Numerical Results \& Discussions}

In this section, we first present results for 2D shock-boundary layer interaction (SBLI) at two Reynolds numbers, namely, 200 and 1000 over both smooth and rough walls. Numerical simulation of 3D SBLI is then conducted. The interaction between shocks and 3D vortical structures are quantified. The numerical setup is stated as follows.

For 2D simulation, the computational domain is chosen as $[0,1] \times[0,0.5]$. Two sets of meshes with uniform mesh sizes, namely, 0.004 and 0.002 , are adopted in 2D simulation. For 3D simulation, the computational domain is chosen as $[0,1] \times[0,0.5] \times[0,0.1]$. To save computational cost, a non-uniform $250 \times 125 \times 12$ mesh with element clustering near the walls is used, as shown in Figure 2(a). The minimum element size in the normal direction of the wall is 0.002 . Boundary conditions for the $3 \mathrm{D}$ simulation are presented in Figure 2(b). The two boundaries in $\mathrm{x}$ direction are set as walls, and the bottom boundary in y direction is also set as wall. The top boundary in y direction is set as symmetry. The two boundaries in $\mathrm{z}$ direction are periodic. The boundary conditions for $2 \mathrm{D}$ simulation are exactly the same as those for 3D simulation, except that there is no need to enforce boundary conditions in $\mathrm{z}$ direction.

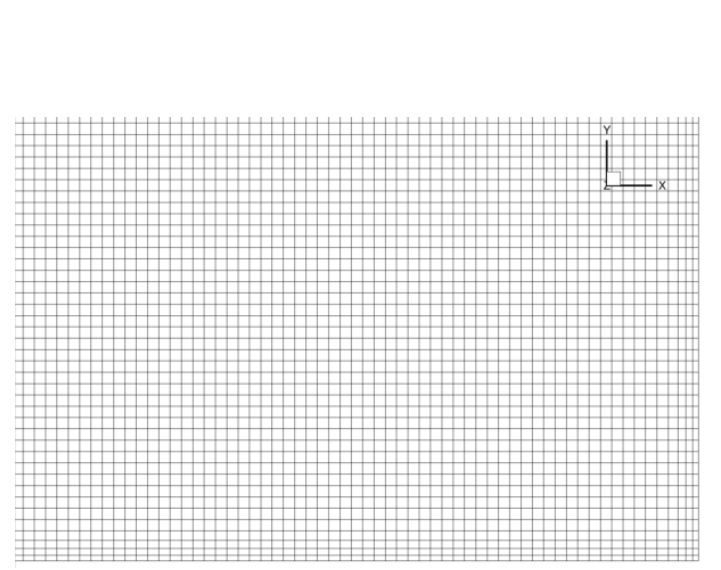

(a)

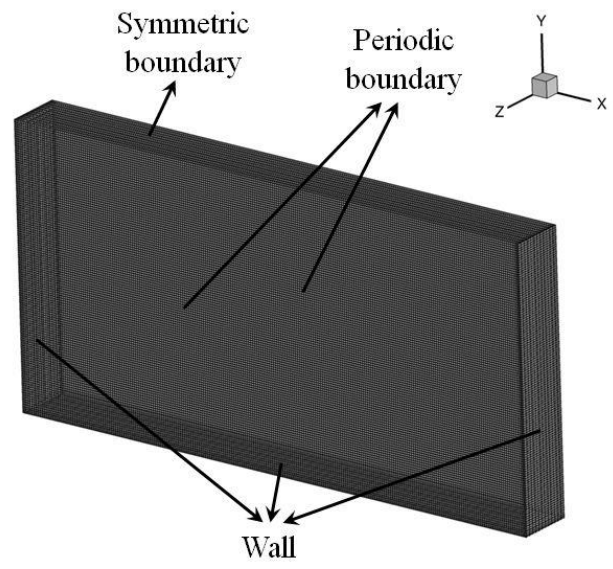

(b)

Figure 2. (a) An enlarged view of the non-uniform $250 \times 125 \times 12$ mesh near the bottom and side walls; (b) setup of boundary conditions for the 3D simulation.

The initial states for the 3D shock-boundary layer interaction are specified as follows:

$$
\begin{cases}\left(\rho_{L}, u_{L}, v_{L}, w_{L}, p_{L}\right)=(120,0,0,0,120 / \gamma) & \text { if } x \leq 0.5 \\ \left(\rho_{R}, u_{R}, v_{R}, w_{R}, p_{R}\right)=(1.2,0,0,0,1.2 / \gamma) & \text { if } x>0.5\end{cases}
$$


The initial states for 2D simulation are the same as those for 3D simulation, except that no velocity $w_{L}$ or $w_{R}$ needs to be given. Similar to the 1D Sod shock tube problem, in this viscous shock tube problem, a shock wave of Mach number 2.37, followed by a contact discontinuity, moves towards the low-pressure region; at the same time, a rarefaction wave moves towards the high-pressure region. After the shock is reflected by the end wall, it will interact with the boundary layer developed when the flow passed over the bottom wall.

\section{III.1. 2D Shock-Boundary Layer Interaction}

\section{III.1.1. Shock-Boundary Layer Interaction over the Smooth Wall at $\mathrm{Re}=200$}

Contours of density and temperature of the 2D shock-boundary layer interaction at $R e=200$ are presented in Figure 3. A coarse mesh (i.e., $500 \times 250$ elements) is used in the FR/CPR simulation, whereas a $4000 \times 2000$ mesh is used in the reference finite difference results [27]. Here note that the degrees of freedom (DOFs) for the $3^{\text {rd }}$-order simulation are $1,125,000$, about $14 \%$ of that of the reference results. Reasonable agreement has been achieved between the current $3^{\text {rd }}$-order simulation and the reference.
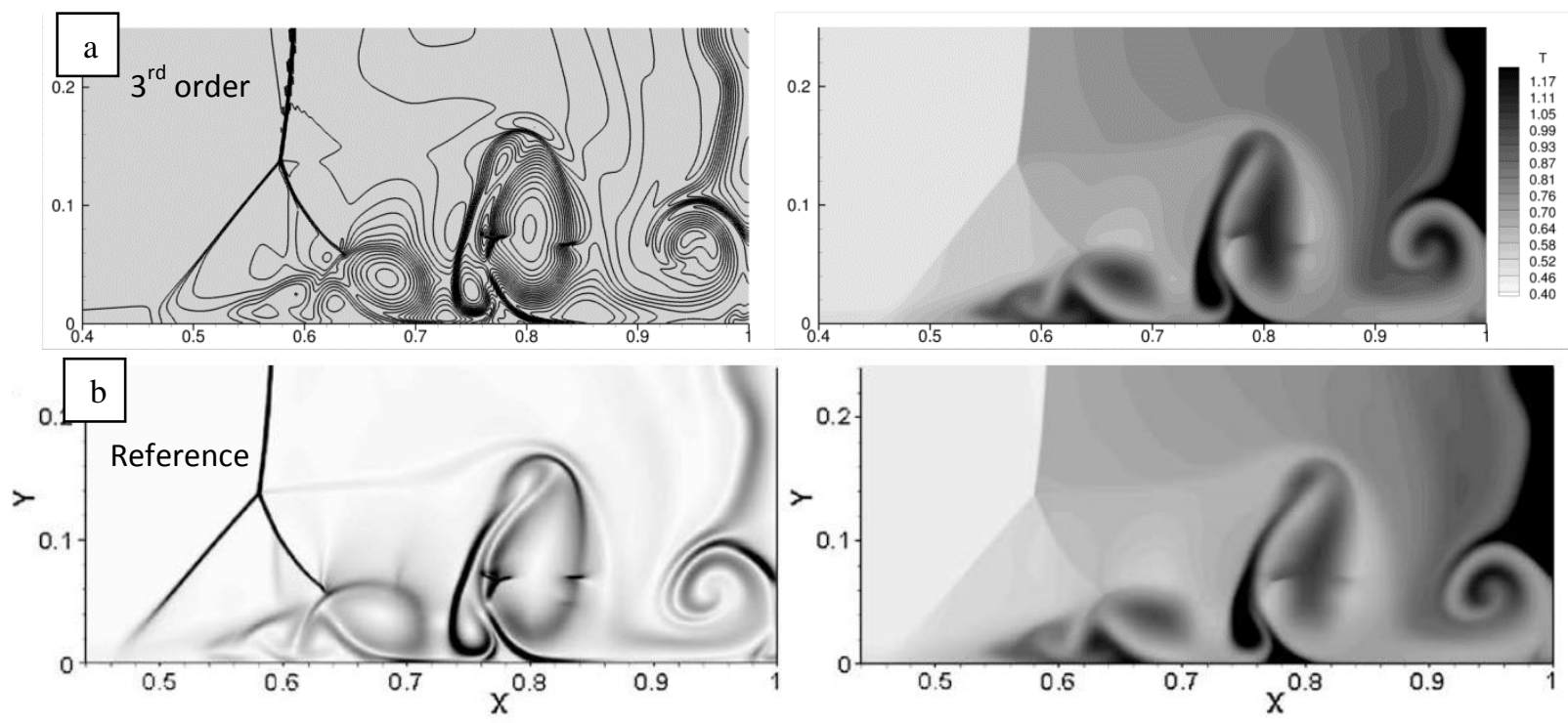

Figure 3. Contours of density (left) and temperature (right) at $t=1$ for $R e=200$. (a) Simulation results using the FR/CPR solver on a $500 \times 250$ mesh with $3^{\text {rd }}$ order of accuracy; (b) Reference results from a $7^{\text {th }}$ order finite difference method on a $4000 \times 2000$ mesh. Note that $|\nabla \rho|$ is used in the reference results. The same contour level for the temperature distribution is used in this figure.

\section{III.1.2. Shock-Boundary Layer Interaction over the Smooth Wall at $\operatorname{Re}=1,000$}

Simulation results of the 2D shock-boundary layer interaction at $R e=1,000$ are presented in Figure 4 , and compared with reference results from literatures. It should be noted that a very coarse mesh (i.e., $250 \times 125$ elements) is used in the FR/CPR simulation, whereas a $4000 \times 2000$ mesh is used in the reference finite difference results [27]. The DOFs for the $3^{\text {rd }}$-order simulation are 281,250 , about $3.5 \%$ of that of the reference results; the DOFs for the $4^{\text {th }}$-order simulation are 500,000 , about $6 \%$ of that of the reference results; the DOFs for the $5^{\text {th }}$-order simulation are 781,250 , about $10 \%$ of that of the reference results; and the DOFs for the $6^{\text {th }}$-order simulation are $1,125,000$, about $14 \%$ of that of the reference 
results. It is found that even with much less degrees of freedoms, the results from the $6^{\text {th }}$-order simulation are quite similar to the reference solution.
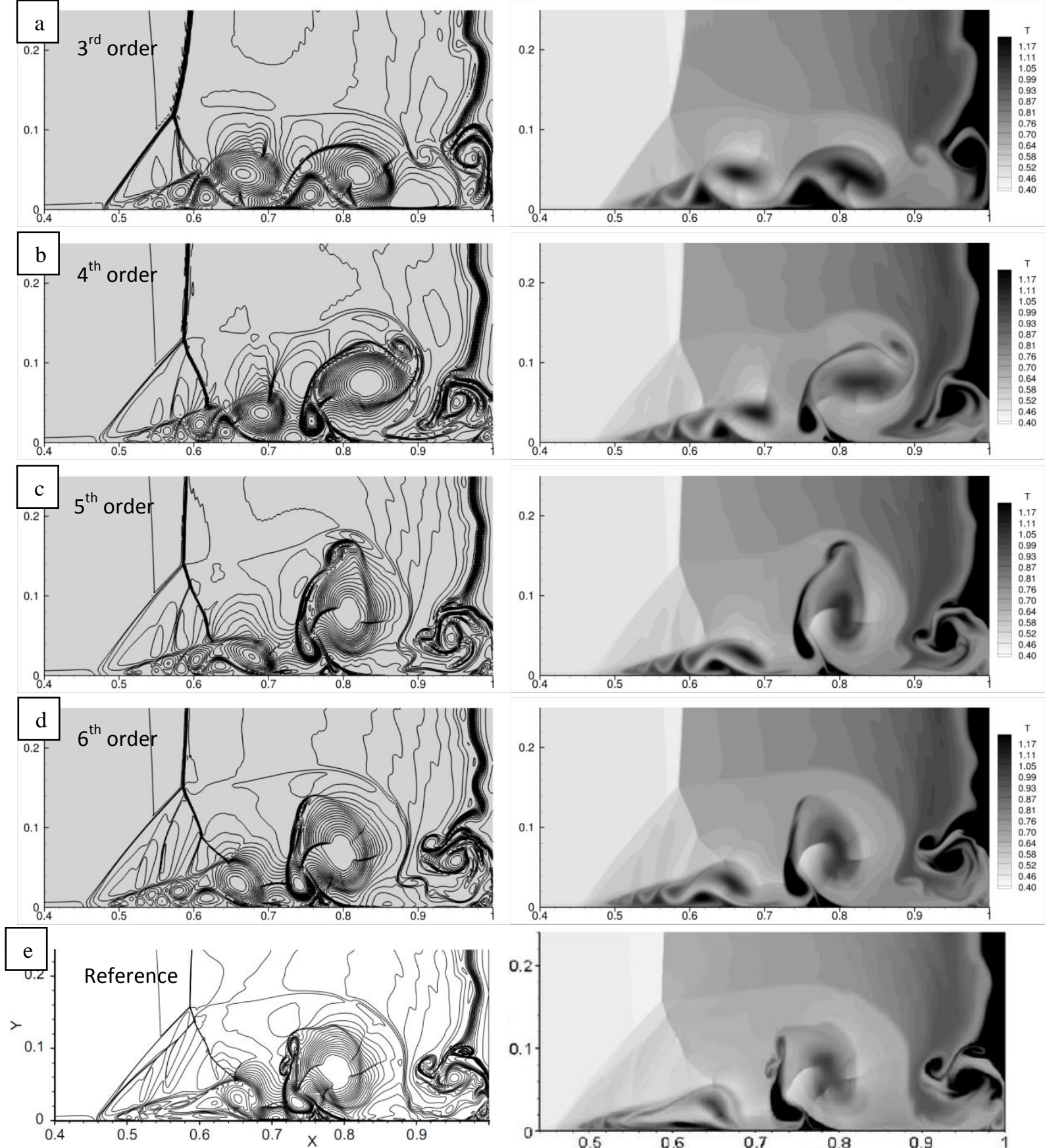

Figure 4. Contours of density (left) and temperature (right) at $t=1$ for $R e=1,000$. (a) (d) are simulated using the FR/CPR solver on a $250 \times 125$ mesh with $3^{\text {rd }}, 4^{\text {th }}, 5^{\text {th }}$ and $6^{\text {th }}$ order of accuracy, respectively; (e) is the reference results from a $7^{\text {th }}$ order finite difference method on a $4000 \times 2000$ mesh. The same contour level is used in this figure.

\section{III.1.3. Shock-Boundary Layer Interaction over the Rough Wall at $\mathrm{Re}=1,000$}

Hypersonic boundary layer flow can be very sensitive to surface roughness near shock-boundary layer interaction region. Three types of the roughness, namely, triangular roughness elements, rectangular 
roughness elements, and rectangular dimple, are studied in this section, as shown in Figure 5. The sizes of these elements are characterized by the height $H$ and width $D$. In all cases, the width $D$ is fixed at 0.032 . Several values of the height $H$ are tested, including 0.001, 0.002. 0.004, 0.008 and 0.016. To distinguish these cases, there are labeled as 'H1', 'H2', 'H3', 'H4' and 'H5', respectively. In this section, all simulations for the $2 \mathrm{D}$ SBLI are performed using the $P^{5}$ (i.e., $6^{\text {th }}$ order) reconstruction.

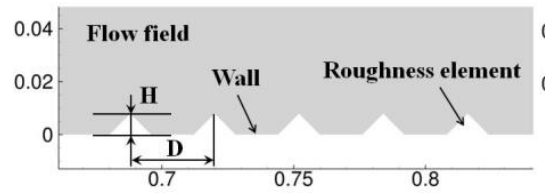

(a)

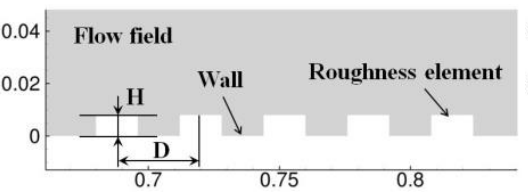

(b)

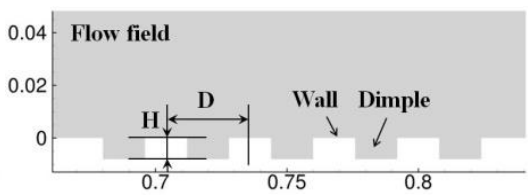

(c)

Figure 5. Configuration of different roughness elements. (a) Triangular roughness elements; (b) rectangular roughness elements; (c) rectangular dimples.

\section{III.1.3.1. Triangular Roughness Elements}

In this test, an array of triangular wall roughness is placed in the boundary layer. These roughness elements can emanate both compression and expansion waves into the flow when shock waves pass over them. As a result, the structures of both shock waves and vortices generated due to shock-boundary layer interaction are changed. Comparing the results in Figure 6 with those in Figure 4, we note that when the height of roughness elements gets larger, the structures of near-wall shocklets and vortices are gradually modified. This can substantially affect the surface heat transfer process.
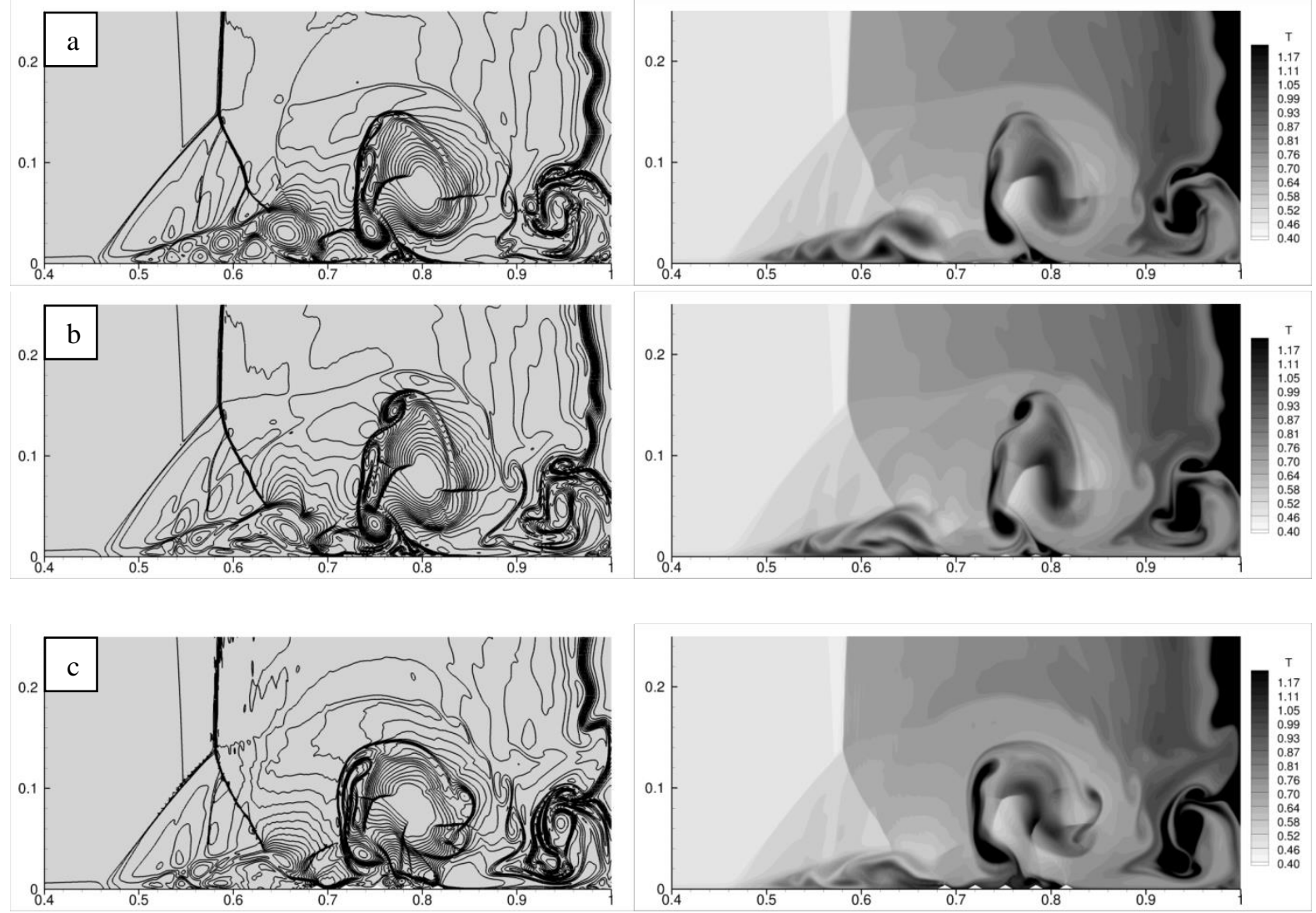

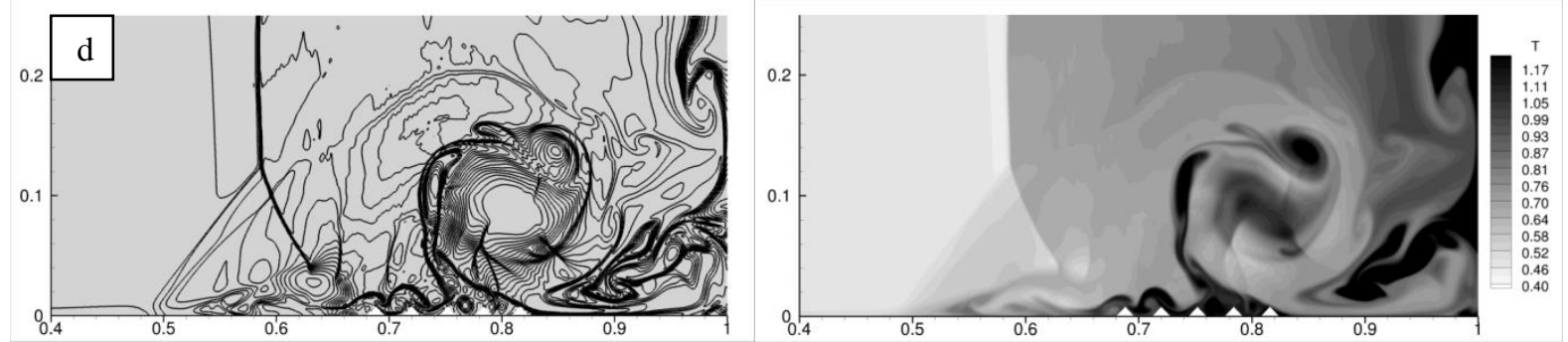

Figure 6. Contours of density (left) and temperature (right) at $t=1$ for $R e=1,000$ with triangular roughness elements of different heights. (a) $H=0.001$; (b) $H=0.002$; (c) $H=0.004$; and (d) $H=0.008$. All cases are simulated using the FR/CPR solver on a $250 \times 125$ mesh with $6^{\text {th }}$ order of accuracy.

The distribution of temperature on the bottom wall with or without triangular roughness elements at $t=1$ is presented in Figure 7. The overall view of wall temperature distribution is displayed in Figure 7 (a) and a detailed view near the region with wall roughness of different sizes (i.e., $x \in[0.68,0.824]$ ) is shown in Figure 7(b). It is observed that wall temperature in the roughness region becomes more uniform as the height of the triangular roughness element becomes larger. By checking the temperature contours shown in Figure 6, it is found that when the height of the roughness element is large enough (e.g., $\mathrm{H}=0.004$ and 0.008 ), the roughness element can substantially affect the evolution of the vortex generated due to the interaction between the shock and the boundary layer. In this case, relatively high triangular elements can enhance the mixing process in the boundary layer. As a result, wall temperature becomes more uniform.

It is also observed that in the region around $x=0.5$ next to the region with high roughness elements, the temperature is lower than that on the smooth wall. This can be explained as follows. Due to the enhanced mixing process in the region with wall roughness, more fluid from the main stream can be dragged down towards the wall in the nearby regions. As presented in the temperature contour from Figure 6 , the fluid in the main stream of the region around $x=0.5$ is colder than that near the wall boundary. It can mix with the fluid near the wall, and reduce the wall temperature.

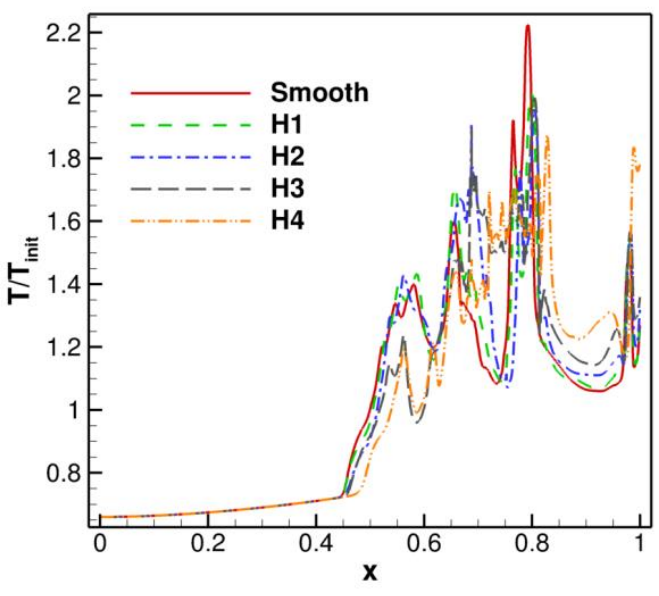

(a)

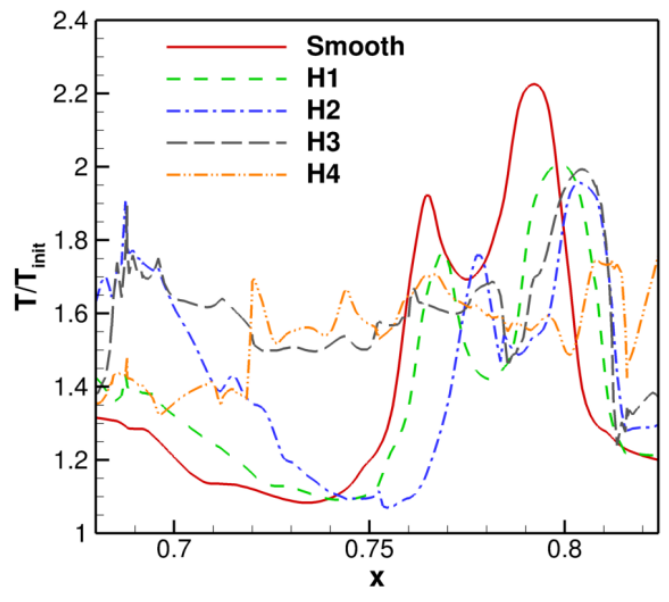

(b)

Figure 7. Temperature distribution on the bottom wall with and without triangular roughness elements at $t=1$ for $R e=1,000$. (a) A complete view; (b) an enlarged view near the region with wall roughness. 


\section{III.1.3.2. Rectangular Roughness Elements}

In this test, an array of rectangular wall roughness is placed in the boundary layer. The height of the wall roughness is fixed at 0.008 (i.e., H4). The density and temperature fields at $t=1$ are presented in Figure 8. The corresponding surface temperature distribution is presented in Figure 9. Similar to the triangular roughness element, the presence of rectangular roughness elements can also enhance the mixing process in the boundary layer. In the region around $x=0.5$ next to the region with roughness elements, the wall temperature also decreases comparing with that over the smooth wall as in the triangular roughness element case. It is also observed that although the vortex structures over rectangular roughness elements are very different from that over triangular roughness elements, the shock structures in the main stream for both cases are very similar.
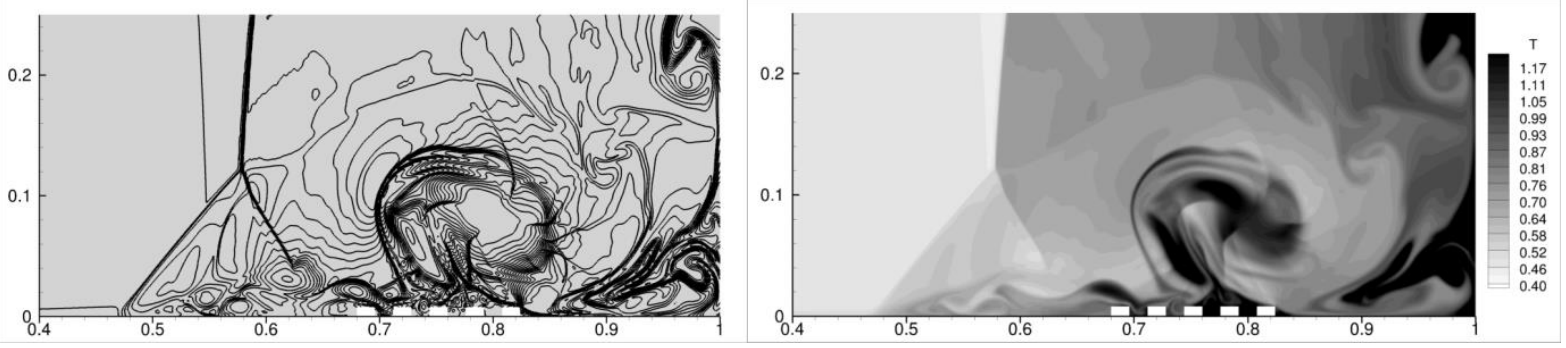

Figure 8. Contours of density (left) and temperature (right) at $t=1$ for $R e=1,000$ with rectangular roughness elements of height $H=0.008$. This case is simulated using the FR/CPR solver on a $250 \times 125$ mesh with $6^{\text {th }}$ order of accuracy.

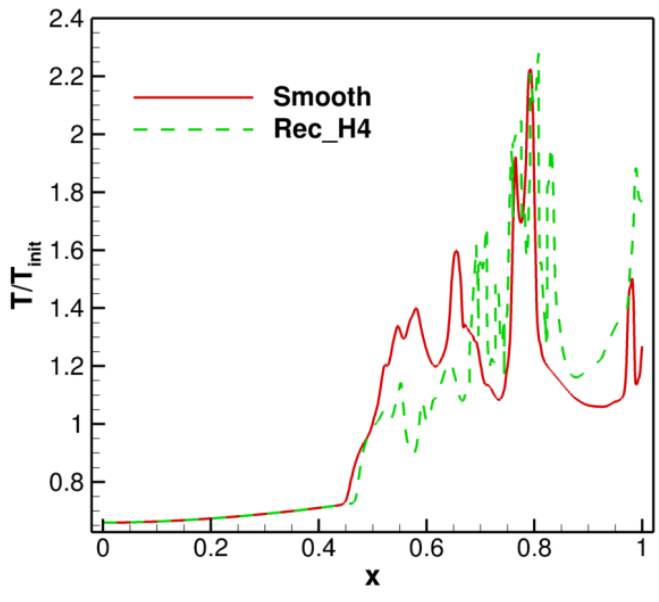

(a)

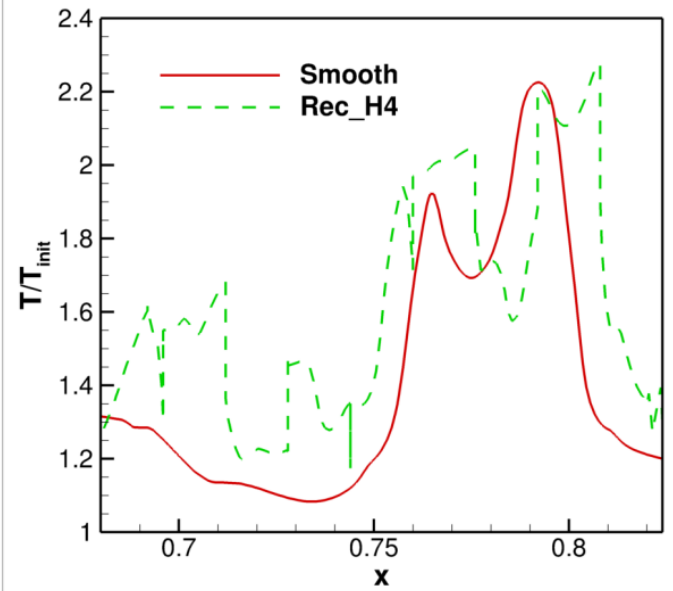

(b)

Figure 9. Temperature distribution on the bottom wall with and without rectangular roughness elements at $t=1$ for $R e=1,000$. (a) A complete view; (b) an enlarged view near the region with wall roughness.

\section{III.1.3.3. Rectangular Dimples}

In this test, an array of rectangular dimples is placed in the boundary layer. Dimple arrays with two different depths, namely, 0.008 and 0.016 , are tested. The density and temperature fields at $t=1$ are presented in Figure 10. The corresponding surface temperature distribution is presented in Figure 11. Comparing with the triangular and rectangular roughness elements, the rectangular dimple does not 
substantially enhance the mixing in the boundary layer. As observed from the temperature contour in Figure 10, the temperature field in the region around $x=0.5$ is similar to that in the smooth wall case. This indicates that mixing in this region is not greatly affected by the wall dimples. Different from the triangular or rectangular elements, the array of rectangular dimples can impede the movement of the vortex behind the shock waves, as observed from Figure 10. The flow physics behind this phenomenon is still under investigation.
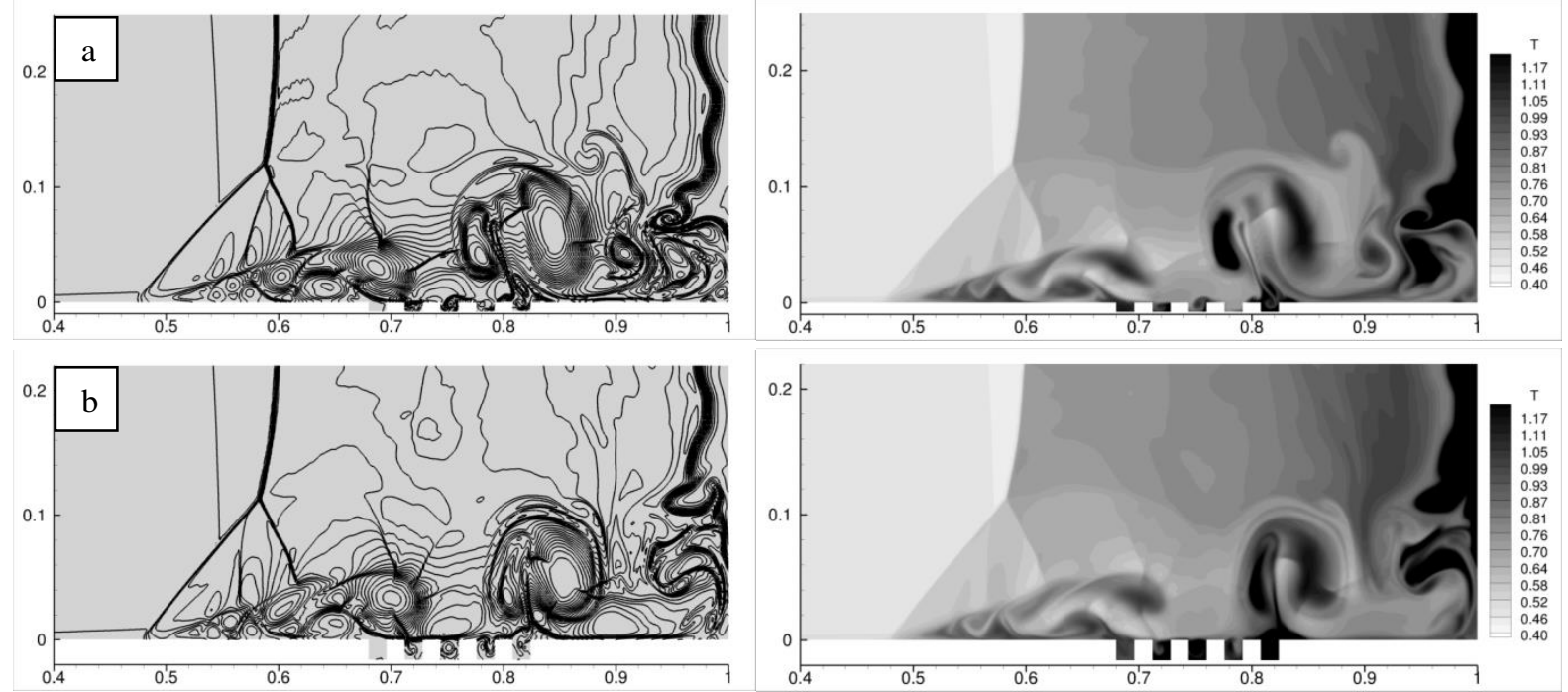

Figure 10. Contours of density (left) and temperature (right) at $t=1$ for $R e=1,000$ with rectangular dimples of different depths. (a) $H=0.008$ and (b) $H=0.016$. All cases are simulated using the FR/CPR solver on a $250 \times$ 125 mesh with $6^{\text {th }}$ order of accuracy.

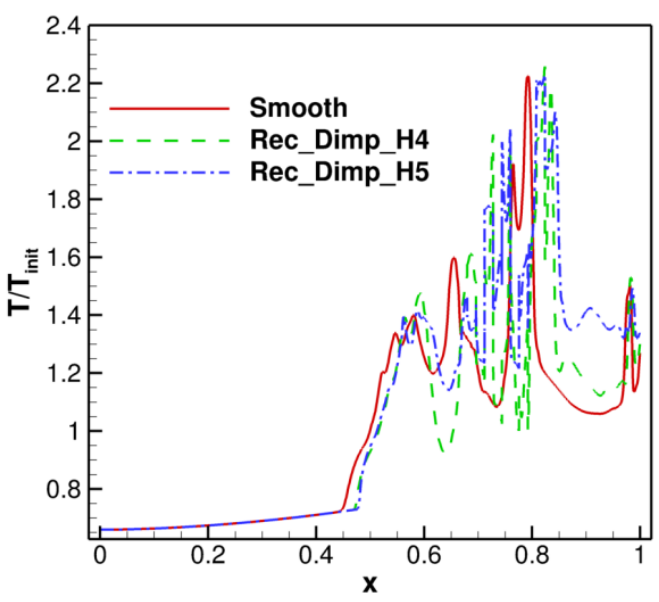

(a)

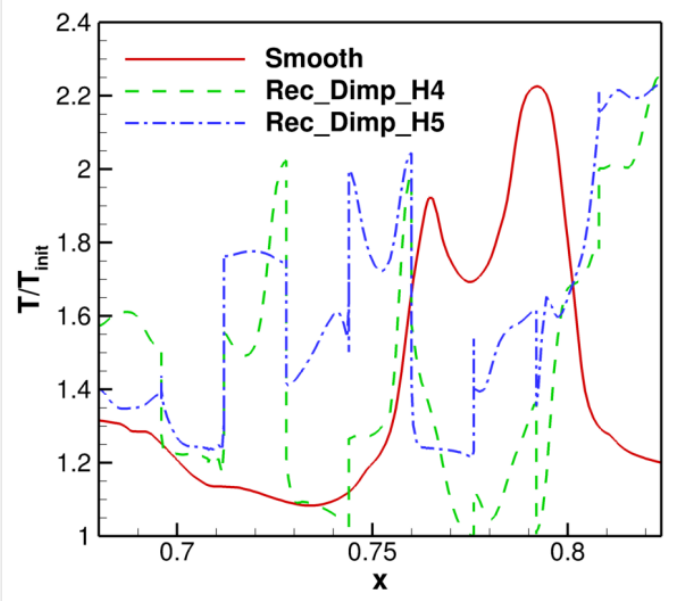

(b)

Figure 11. Temperature distribution on the bottom wall with and without rectangular dimples at $t=1$ for $R e=$ 1,000. (a) A complete view; (b) an enlarged view near the region with wall roughness. 


\section{III.1.3.4. Summary}

To summarize SBLI over the rough wall discussed in this section, the wall temperature distribution with and without wall roughness at $t=1$ is plotted in Figure 12. The roughness size used in this figure is 0.008. In current study, it is found that the triangular roughness element can effectively enhance the mixing process in the boundary layer. The rectangular roughness element functions similarly as the triangular one, although the wall temperature is not as uniform as that of the triangular roughness element after mixing. The rectangular dimple does not greatly enhance the mixing in the boundary layer. From the simulation results, it is also observed that all types of wall roughness can substantially modify the evolution of the vortices originated from SBLI. The temperature fields associated with streamlines near the region with wall roughness are displayed in Figure 13. It is clearly seen from these figures that the vortex structure and thus the wall temperature distribution is very sensitive to the roughness height and shape due to the interaction between the fluid near the boundary and the wall roughness.

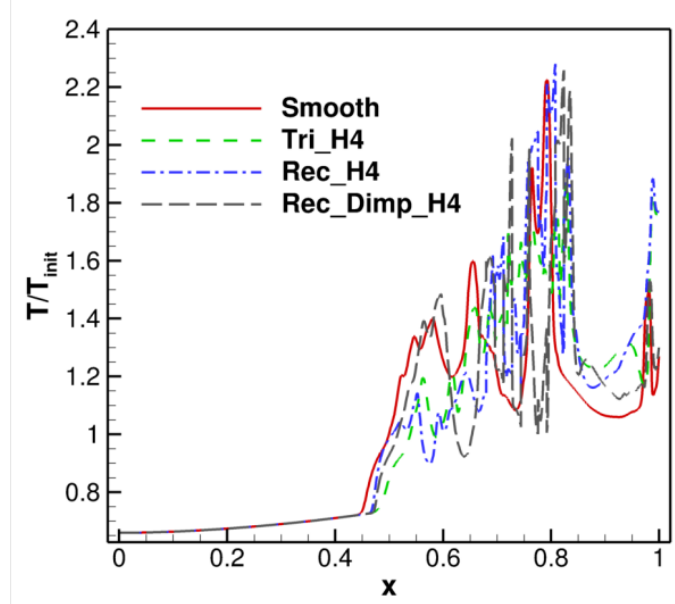

(a)

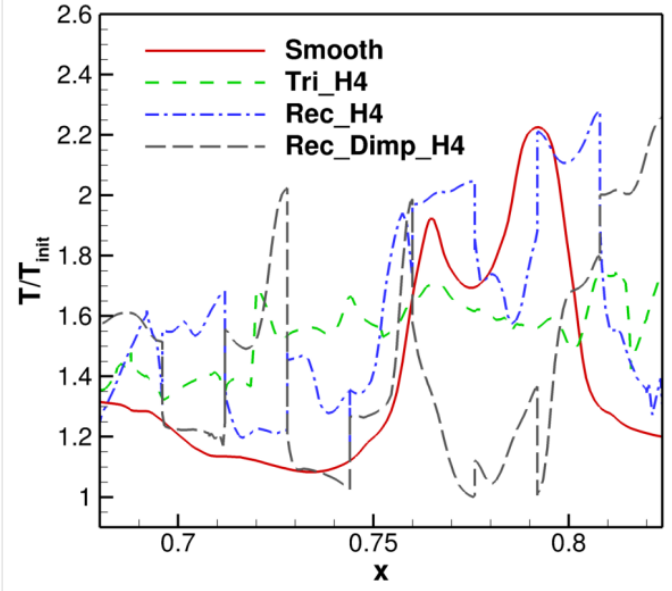

(b)

Figure 12. Temperature distribution on the bottom wall with and without wall roughness at $t=1$ for $R e=1,000$. The element height/depth is set as $H=0.008$. (a) A complete view; (b) an enlarged view near the region with wall roughness.

\section{III.2. 3D Shock-Boundary Layer Interaction}

In this section, the 3D SBLI is simulated at $\operatorname{Re}=200$ and $\mathrm{Re}=1,000$. For the Reynolds number 200 , both case with the smooth wall and that with triangular prism roughness elements are studied. The height of the wall roughness is fixed at 0.008 . For the Reynolds number 1,000, only the case with the smooth wall is simulated in current study. All simulations are performed using the $P^{2}$ (i.e., $3^{\text {rd }}$ order) reconstruction.

The temperature fields for both cases with and without wall roughness are displayed in Figure 14. Temperature on three surfaces is shown, namely, the bottom wall parallel to the $\mathrm{XZ}$ plane, the side wall parallel to the $\mathrm{YZ}$ plane, and the periodic boundary parallel to the $\mathrm{XY}$ plane. It is found that the temperature distribution on the periodic boundary for the case with the smooth wall is very similar to that shown in Figure 3 from the 2D SBLI simulation. In the current case, some turbulent features have shown up in the boundary layer near the bottom wall, which will increase the mixing near this region. This results in more uniform temperature distribution near the bottom wall compared with the 2D simulation. 


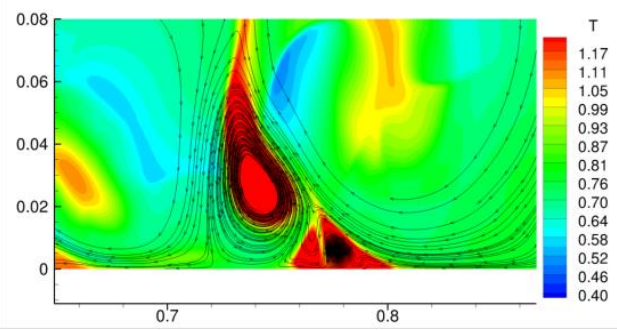

(a)

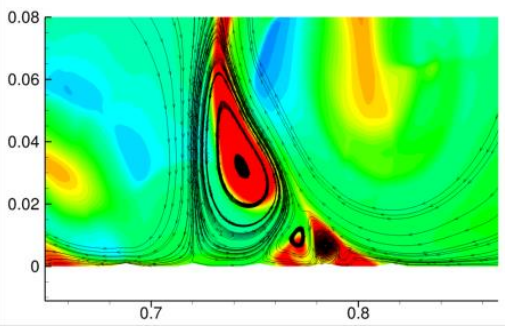

(b)

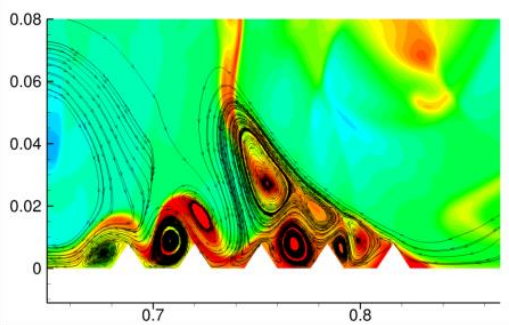

(e)

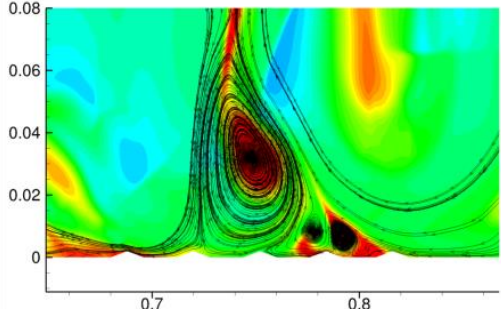

(c)

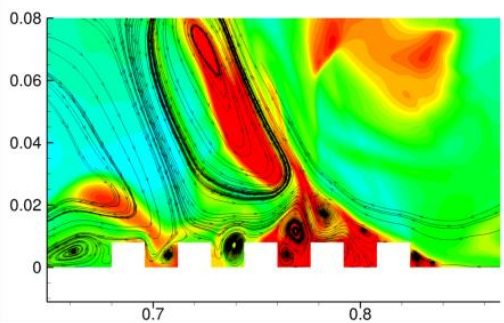

(f)

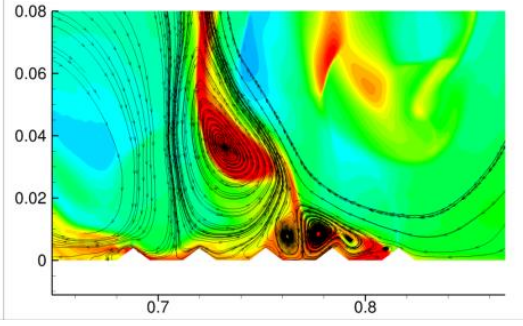

(d)

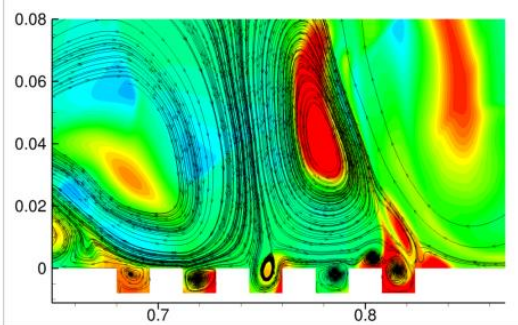

(g)

Figure 13. Streamlines and temperature fields near the region with wall roughness at $t=1$ for $R e=1,000$. (a) Smooth wall; (b) triangular roughness element, $H=0.001$; (c) triangular roughness element, $H=0.002$; (d) triangular roughness element, $H=0.004$; (e) triangular roughness element, $H=0.008$; (f) rectangular roughness element, $H=0.008$; and $(\mathrm{g})$ rectangular dimple, $H=0.008$.

Similar to the conclusion from Section III.1.3., wall roughness can substantially modify the vortex structure originated from SBLI, and thus the wall temperature distribution. Wall roughness has limited effect on the shock structure in the main stream. The vorticity field for the case with wall roughness is displayed in Figure 15. In this figure, the vortex structure is indicated by the Q-criterion, and the density flow on the periodic boundary is also displayed to locate the position of the shock. It is clear that the vortical flow behind shock has started to become turbulent in this case. 


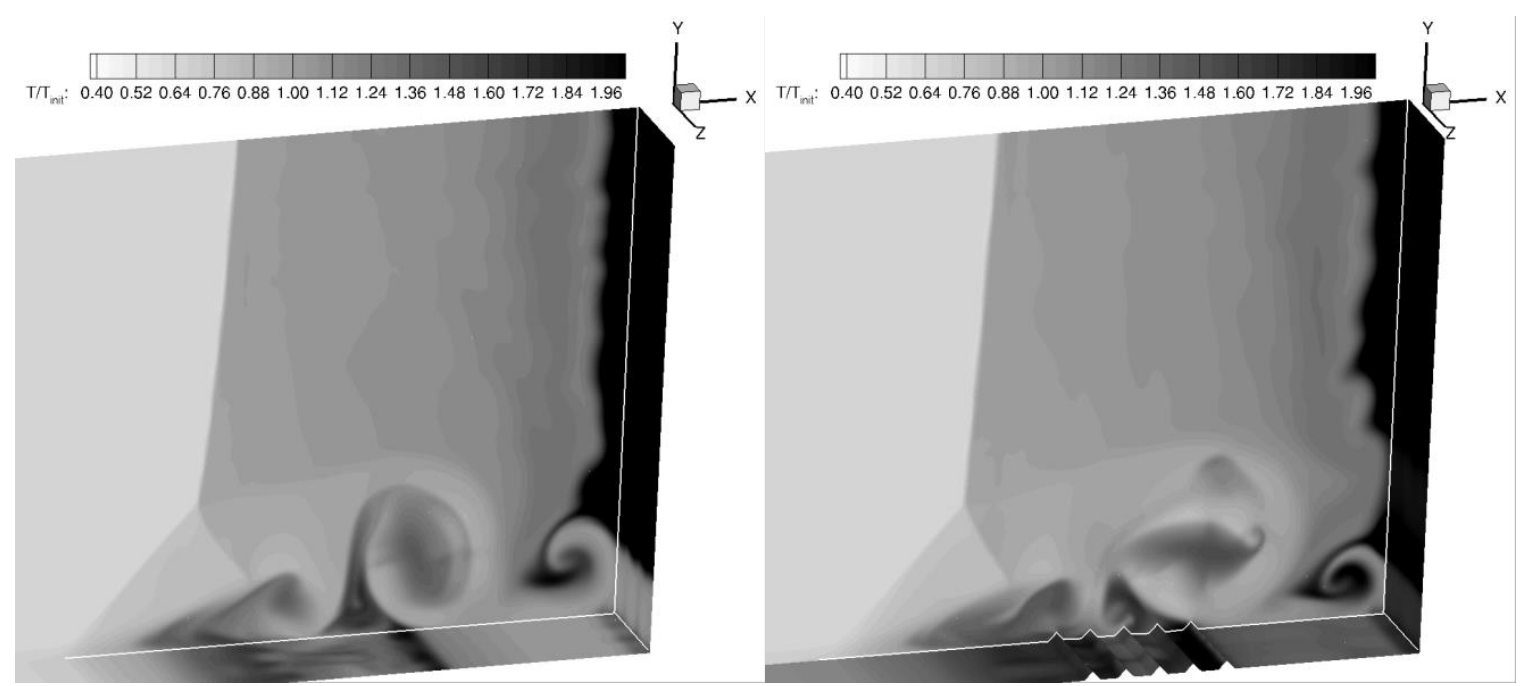

Figure 14. Temperature contours on the bottom wall, side wall and periodic boundary at $t=1$ for $R e=200$ for 3D SBLI. (a) smooth wall and (b) wall with triangular prism roughness elements of height $\mathrm{H}=0.008$. All cases are simulated using the FR/CPR solver on a non-uniform $250 \times 125 \times 12$ mesh with $3^{\text {rd }}$ order of accuracy.

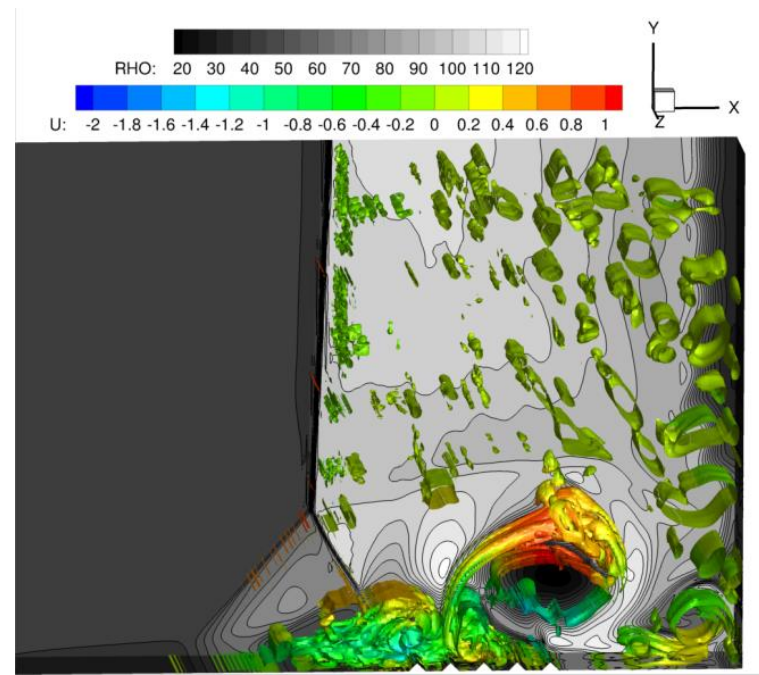

Figure 15. Vorticity field of 3D SBLI over a wall with triangular prism roughness elements of height $\mathrm{H}=0.008$ at $t=1$ for $R e=200$. The vortex structure is indicated by the $\mathrm{Q}$-criterion colored by the streamwise velocity. The background is the density contour on the periodic boundary.

When the Reynolds number increases to 1,000 , the flow behind shock can be fully turbulent with a very short laminar-turbulent transition region. This is clearly seen from the vorticity field as shown in Figure 16(a). The corresponding temperature distribution on the bottom wall, side wall and periodic boundary is displayed in Figure 16(b). It is observed that turbulence can significantly enhance the fluid mixing near wall boundaries. 


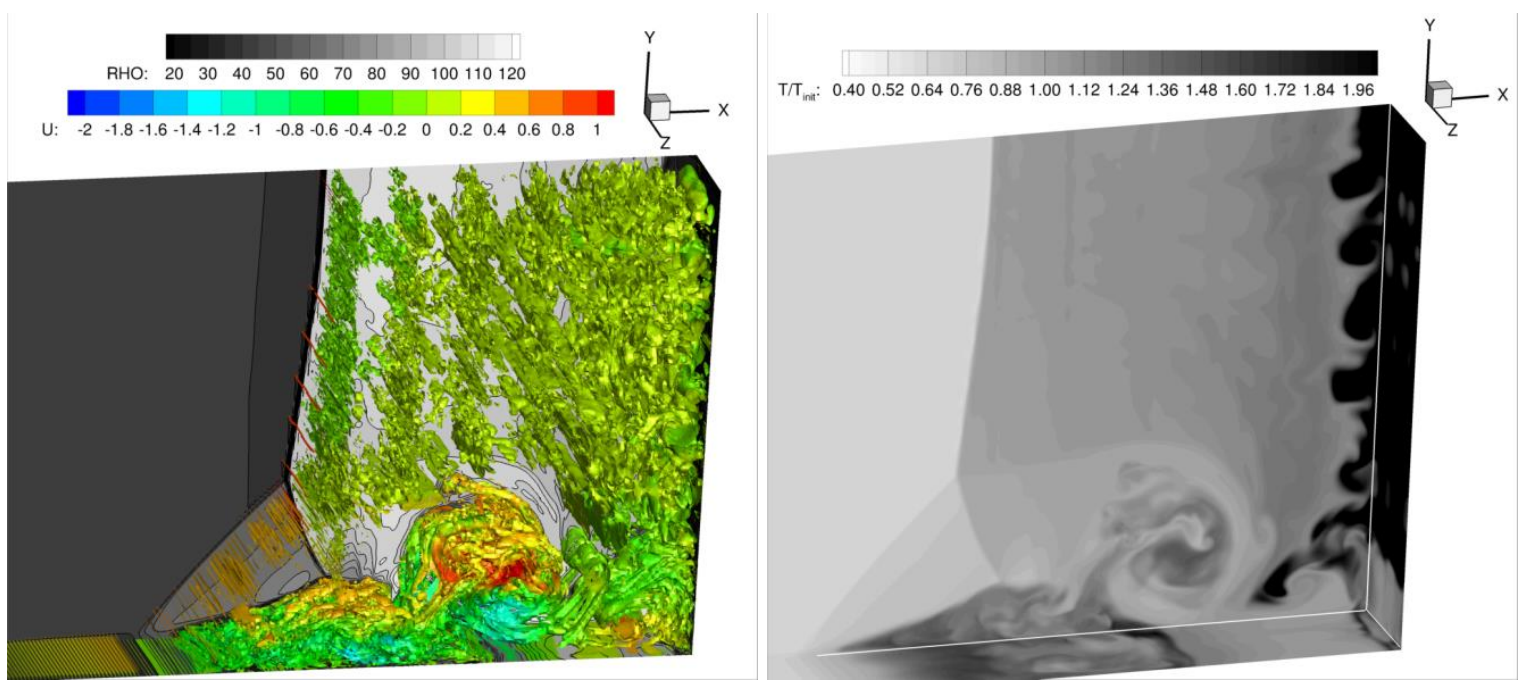

Figure 16. (a) Vorticity field and (b) temperature contours on the bottom wall, side wall and periodic boundary at $t=1$ for $R e=1,000$ for 3D SBLI over a smooth wall. The vortex structure is indicated by the Q-criterion colored by the streamwise velocity. The background in (a) is the density contour on the periodic boundary.

\section{Conclusions}

A high-order accurate FR/CPR method equipped with localized Laplacian artificial viscosity (LLAV) shock-capturing capability is used to simulate shock-boundary layer interaction (SBLI) at different Reynolds numbers with and without wall roughness. It is found that the method can effectively and sharply resolve both shock and boundary-layer separation features.

From the 2D simulation results, it is observed that surface roughness can greatly affect the interaction between the shock waves and the boundary layer. Specifically, all types of wall roughness can change the evolution process of the vortex originated from SBLI. As a result, the surface heat transfer process can be substantially modified. It is also found that the protruding roughness elements (e.g., triangular and rectangular roughness elements in this study) can enhance the mixing in the boundary layer, whereas the dimple-type roughness elements do not show prominent fluid mixing enhancement features.

From the 3D SBLI simulation results, it is observed that the flow can quickly transit from its laminar state to turbulent state in a very short laminar-turbulent transition region behind shock. This will significantly enhance the fluid mixing in the boundary layer, and modify the surface heat transfer. In the future work, more research will be conducted to fully unveil the flow physics in 3D SBLI with wall roughness.

\section{References}

[1] Y. Andreopoulos, J. H. Agui and G. Briassulis, "Shock wave-turbulence interactions," Annu. Rev. Fluid Mech., vol. 32, pp. 309-345, 2000.

[2] D. S. Dolling, "Fifty years of shock-wave/boundary-layer interaction research: what next?," AIAA Journal, vol. 39, no. 8, pp. 1517-1531, 2001.

[3] A. J. Smits, M. P. Martin and S. Girimaji, "Current status of basic research in hypersonic turbulence," in 39th AIAA Fluid Dynamics Conference and Exhibit, Orlando, FL, 2009.

[4] G. V. Candler, D. J. Mavriplis and L. Trevino, "Current status and future prospects for the numerical simulation of hypersonic flows," in 47th AIAA Aerospace Sciences Meeting Including the New 
Horizons Forum and Aerospace Exposition, Orlando, FL, 2009.

[5] P. A. Gnoffo and J. A. White, "Computational aerothermodynamic simulation issues on unstructured grids," in 37th AIAA Thermophysics Conference, Portland, Oregon, 2004.

[6] G. Candler, M. Barnhardt, T. Drayna, I. Nompelis, D. Peterson and P. Subbareddy, "Unstructured grid approaches for accurate aeroheating simulations," in 18th AIAA Computational Fluid Dynamics Conference, Miami, FL, 2007.

[7] H. Huynh, Z. Wang and P. Vincent, "High-order methods for computational fluid dynamics: A brief review of compact differential formulations on unstructured grids," Computers \& Fluids, vol. 98, pp. 209-220, 2014.

[8] P.-O. Persson and J. Peraire, "Sub-Cell Shock Capturing for Discontinuous Galerkin Methods," Reno, Nevada, 2006.

[9] G. E. Barter and D. L. Darmofal, "Shock capturing with PDE-based artificial viscosity for DGFEM: Part I. Formulation," J. Comput. Phys., vol. 229, pp. 1810-1827, 2010.

[10] M. L. Yu, F. X. Giraldo, M. Peng and Z. Wang, "Localized artificial viscosity stabilization of discontinuous Galerkin methods for nonhydrostatic mesoscale atmospheric modeling," Monthly Weather Review, vol. 143, no. 11, pp. 4823-4845, 2015.

[11] B. Cockburn and C.-W. Shu, "TVB Runge-Kutta local projection discontinuous Galerkin finite element method for conservation laws II: general framework," Math. Comput., vol. 52, pp. 411-435, 1989.

[12] F. Bassi and S. Rebay, "High-order accurate discontinuous finite element solution of the 2D Euler equations," J. Comput. Phys., vol. 138, pp. 251-285, 1997.

[13] Z. J. Wang, "Spectral (finite) volume method for conservation laws on unstructured grids: basic formulation," J. Comput. Phys., vol. 178, pp. 210-251, 2002.

[14] Y. Liu, M. Vinokur and Z. J. Wang, "Spectral difference method for unstructured grids I: Basic formulation," J. Comput. Phys, vol. 216, pp. 780-801, 2006.

[15] M. L. Yu, Z. J. Wang and Y. Liu, "On the accuracy and efficiency of discontinuous Galerkin, spectral difference and correction procedure via reconstruction methods," Journal of Computational Physics, vol. 259, pp. 70-95, 2014.

[16] M. L. Yu and Z. Wang, "Shock capturing for the correction procedure via reconstruction method using artificial viscosity and diffusivity," in ICCFD8, Chengdu, China, 2014.

[17] J. S. Park, M. Yu, C. Kim and Z. Wang, "Comparative study of shock-capturing methods for highorder CPR: MLP and artificial viscosity," in ICCFD8, Chengdu, China, 2014.

[18] P. L. Roe, "Approximate riemann solvers, parameter vectors and difference schemes," Journal of Computational Physics, vol. 43, pp. 357-372, 1981.

[19] F. Bassi and S. Rebay, "GMRES Discontinuous Galerkin Solution of the Compressible NavierStokes Equations," in Discontinuous Galerkin Methods - Theoriy, Computation and Application, Springer, 2000, pp. 197-208.

[20] H. T. Huynh, "A flux reconstruction approach to high-order schemes including discontinuous Galerkin methods," in the 18th AIAA Computational Fluid Dynamics Conference, Miami, FL, 2007.

[21] M. L. Yu and Z. J. Wang, "On the connection between the correction and weighting functions in the correction procedure via reconstruction method," J. Sci. Comput., vol. 54, no. 1, pp. 227-244, 2013.

[22] Z. J. Wang and H. Y. Gao, "A unifying lifting collocation penalty formulation for the Euler equations," AIAA Paper 2009-401.

[23] P. E. Vincent, P. Castonguay and A. Jameson, "Insights from von Neumann analysis of high-order flux reconstruction schemes," J. Comput. Phys. 230, pp. 8134-8154, 2011.

[24] Z. J. Wang, Adaptive high-order methods in computational fluid dynamics, Advances in 
computational fluid dynamics Vol.2, 2011.

[25] Z. Wang, K. Fidkowski, R. Abgrall, F. Bassi, D. Caraeni, A. Cary, H. Deconinck, R. Hartmann, K. Hillewaert, H. Huynh, N. Kroll, G. May, P.-O. Persson, B. v. Leer and M. Visbal, "High-order CFD methods: current status and perspective," International Journal for Numerical Methods in Fluids, vol. 00, pp. 1-42, 2012.

[26] A. Mani, J. Larsson and P. Moin, "Suitability of artificial bulk viscosity for large-eddy simulation of turbulent flows with shocks," Journal of Computational Physics, vol. 228, no. 19, pp. 7368-7374, 2009.

[27] V. Daru and C. Tenaud, "Numerical simulation of the viscous shock tube problem by using a high resolution monotonicity-preserving scheme," Comput. Fluids, vol. 38, pp. 664-676, 2009. 